\title{
Let the Past Rest: The Influence of Effortful Control on Directed Forgetting of Explicit and Implicit Memories
}

\author{
Yang Yang ${ }^{1, *, \dagger}$, Lixing Yin ${ }^{2, \dagger}$, Jinghan You $^{3, \dagger}$, Zichen Zhang ${ }^{4, \dagger}$ \\ ${ }^{1}$ Queen Mary University of London, London, UK \\ ${ }^{2}$ Southwest University, Chongqing, China \\ ${ }^{3}$ School of Continuing Education, SJTU, Shanghai, China \\ ${ }^{4}$ University of British Columbia, Vancouver, BC, Canada \\ ${ }^{*}$ Corresponding author.Email:ml201143@qmul.ac.uk \\ $\dagger$ These authors contributed equally.
}

\begin{abstract}
The ability of directed forgetting is closely related to the individuals' level of effortful control. This study aims to investigate the effect effortful control has on the level of directed forgetting. Forty-nine Chinese participants of ages between 18 and 25 were invited to this study. Participants were selected according to their level of effortful control, and they were divided into four groups, with each group doing different memory tests. Afterward, they were presented with Chinese characters that are less common, followed by an interference task. In the next stage, participants were tested with a recognition task or perceptual identification task. In explicit memory, there was an interaction effect between cues and participants' performances in memory tasks $(p<0.05)$, while in implicit memory, no such effect was observed $(p>0.05)$. The findings suggest that although directed forgetting exists in both explicit and implicit memory, the level of effortful control does not affect implicit memory, and in explicit memory, individuals with a higher level of effortful control show the better ability of directed forgetting This study provides evidence that there is a relationship between temperament and memory.
\end{abstract}

Keywords: Directed forgetting, Effortful control, Implicit memory, Explicit memory.

\section{INTRODUCTION}

Directed forgetting (DF) is an experimental procedure developed in the late 1960s as an analogue to normal updating of memory [1]. DF refers to the intentional and cognitive forgetting of memorized information [2]. The research paradigm of directed forgetting, on the other hand, was first formally proposed by Bjork in 1972. Bjork suggested that to measure directed forgetting, the subjects were first presented with learning materials, with part of which followed by a cue to remember $(\mathrm{R})$ and the rest to forget (F). The level of directed forgetting was estimated according to the effect of instructions on subjects' performances in the final memory test, and there are two methods to evaluate it: the item method and the list method. In the item method, learning materials are presented one by one, each followed by a cue of remembering and forgetting, while in the list method, the instruction is given after the presenting of an entire list of learning materials [3]. In some studies, the item method was tied to attentional control and might include inhibitory mechanisms. Previous research using the DF paradigm confirmed that people have worse performances on recognizing tasks of the items followed by a cue to forget $(\mathrm{F})$ than a cue to remember $(\mathrm{R})$. This suggests that it is possible to voluntarily control our cognition to impair access to unwanted information [4], which might be a possible solution to intrusive memory.

For decades, increasing empirical research has recognized intrusive memory as a common feature of many mental disorders [5]. Patients with post-traumatic stress disorder, for instance, are suffering from a variety of symptoms, including re-experiencing, avoidance, negative cognition and mood, and hyperarousal [6]. In clinical psychology, one traditional approach to treating this disorder is visual intrusions, which were noted to be repetitive, uncontrollable, and distressing [5]. In this context, one possible outlet might be to make the survivors forget the traumatic memories. 
The human brain has a remarkable capacity to acquire, store, and recall information over a long period of time [7]. On the other hand, forgetting is an adaptive feature of memory that helps to improve access to other memories by discarding unwanted or irrelevant information. The brain automatically decides which information should be stored and which discarded, and this enables people to exert volitional control over what they forget. Previous research has associated the intentional forgetting of new memories with increased activity in the right dorsolateral prefrontal cortex and decreased activity in the hippocampus, along with increased functional coupling between these two regions [8].

Although much research has been done in this field, there is no clear information on how exactly humans could choose what to forget and what to remember. In other words, make the process of forgetting intentional. Under this circumstance, we found that intentional forgetting was mentioned to be an effortful process [9]. We, therefore, suspect that effortful control [4] might be an influencing factor to intentional forgetting, and to go further, DF.

First proposed by Rothbart in 2001, EC was defined as an attention-related temperament dimension reflecting the individual difference in emotional and behavioural regulation, through factor analysis of children's behavioural questionnaires [10]. EC is generally believed to be the result of the development of executive attention which can be measured through selfreport or other-report questionnaires [10]. The concept of EC includes three aspects: attention control, inhibition control and activation control [11]. EC is a component of temperament associated with selfregulation and executive function. Referred to a series of cognitive processes that control behaviour including attentional control, cognitive inhibition, inhibitory control, working memory, and cognitive flexibility, executive function is considered to relate to directed forgetting closely [12].

Despite all the information, it is still not clear whether there exists DF, with few studies that have mentioned what exactly affects the level of DF. Moreover, there are barely any studies investigating the phenomenon of directed forgetting in implicit memory. Therefore, the aim is to investigate whether DF exists and what its influence factors are. Several possible factors were analysed and investigated further the relationship between effortful control [4] and directed forgetting. We have made two research hypotheses. First, there exists directed forgetting. Second, people with a higher level of effortful control would be more abled in directed forgetting of explicit and implicit memories. it could be expected that people with a higher ability of effortful control would have better inhibitory control ability, which leads to a greater effect of directed forgetting.

\section{METHOD}

\subsection{Participants}

Forty-nine participants between 18 and 25 years of age (12 male, 37 female, Mage $=20.73, \mathrm{SD}=1.27)$ took part in the study. They $(\mathrm{N}=210)$ were recruited via online advertising. Participants whose scores were in the top $27 \%$ and bottom $27 \%$ of the EC subtest of the Adult Temperament Questionnaire (ATQ) [12] were invited to participate in the study. One question was added to the original ATQ, which requires the subjects to choose a certain option in order to test the random clicking. In addition, subjects whose scores are higher or lower than 2.5 standard deviations above the mean were also excluded. Finally, 19 high EC participants (8 male) and 30 low EC participants (4 male) completed the DF memory task. All reported normal or corrected normal vision. Participants were compensated $¥ 5$ for attending. The study was approved by the local ethics committee. See Fig. 1 for the screening process.

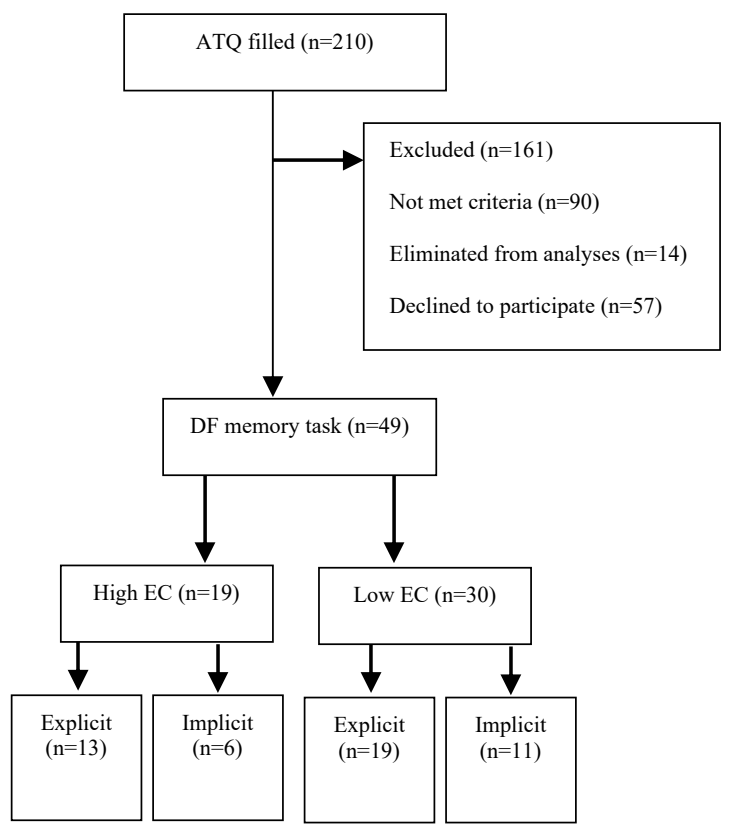

Figure 1 Participant selection chart. Explicit or Implicit represent the explicit or implicit memory test.

\subsection{Materials}

ATQ: The Adult Temperament Questionnaire (ATQ) was adapted from the Physiological Reactions Questionnaire [13], including general constructs of effortful control, negative affect, extraversion/surgency, and orienting sensitivity [8]. The ATQ standard form includes 177 items [12]. The EC subscale of the Chinese version of the ATQ was used, including 35 items for assessing inhibitory control, activation control and attentional control [12]. The alpha reliability was 0.75 
[12]. The questionnaire used a 7-point Likert scale $(1=$ very inconsistent, $7=$ very consistent), in which 21 questions were scored in reverse. Subjects with a score less than or equal to 4 were classified as LEC group, and those with a score greater than or equal to 4.58 were classified as HEC group.

Chinese characters: Ninety-two Chinese characters were selected from the Less Common Character List from the Contemporary Chinese Dictionary (https://www.zdic.net/zd/zb/cc2/). The number of strokes and the emotionality of Chinese characters are controlled during selection: 1) Characters with ten to fifteen strokes in the list of low-frequency characters are used; 2) Controlling emotionality means that a character has no obvious positive or negative meaning and it's not an emotional character.

\subsection{Procedure}

The experiment was conducted online with the help of the Pavlovia platform. Participants were connected to the experimenter by video call on WeChat during the experiment. They were informed to prepare the computer with a keyboard beforehand. Stimulus presentation and response collection were controlled by experimental software written in Psychopy3.

After giving informed consent, participants were instructed to view a series of study trials in which each character would be followed by a Remember (R) or Forget (F) instruction. The experiments lasted for less than 20 minutes, including three phases: 1) Learning phase. Participants were told that if they received an $\mathrm{R}$ instruction, they were to remember the character and that if they received an $\mathrm{F}$ instruction, they were to forget the character. 2) Interference phase. 3) Testing phase. A recognition test is used for explicit memory, and perceptual identification is used for implicit memory.

Learning phase: As depicted in Figure 2, each trial in the study phase began with the presentation of a centralized stimulus ("+") lasting 1 sec. A Chinese character for $3 \mathrm{sec}$ replaced fixation. After that, an R or $\mathrm{F}$ memory cue was assigned to each character. Individual characters were presented randomly without replacement. In addition. four toy trials were designed for practice before the learning stage.

Interference phase: This phase aims to mask the relationship between learning and testing. Participants were instructed to count backwards from 1000 and minus 7 each time for 2 minutes.

Testing phase: Following the interference phase, the experiment advanced to the testing phase, during which participants were presented with the $22 \mathrm{R}$ and $22 \mathrm{~F}$ characters from the study trials, intermixed randomly with 44 foil characters that had not been presented in the study phase. Participants were instructed to press " $F$ " on the computer keyboard to indicate a character that had been presented previously (i.e., $\mathrm{R}$ and $\mathrm{F}$ characters) or "J" to indicate a character that had not been presented (i.e., foil characters). For explicit memory, participants were allowed to respond to this task at their own pace whereas the characters for the implicit memory test were only shown for $30 \mathrm{~ms}$.

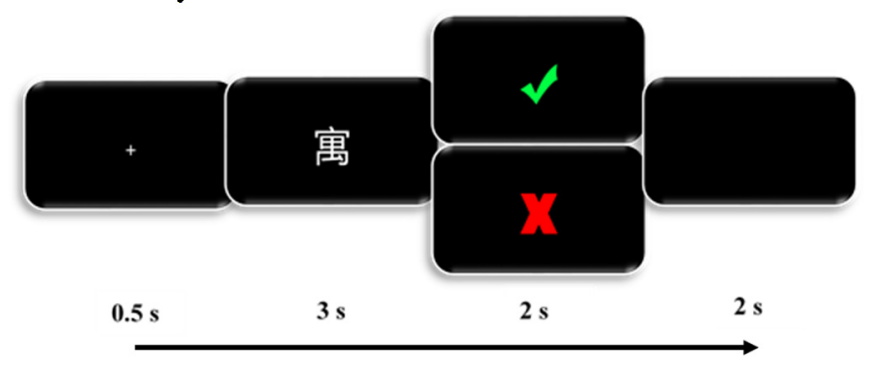

Figure 2 Trials in the study phase. Green check is the cue for remembering and the red cross is the cue for forgetting. The Chinese character in the demonstration is pronounced "Yu" in Chinese Pinyin, which means to live or contain.

\subsection{Data analysis}

The threshold for statistical significance was set at $\mathrm{p}$ $<0.05$ for all analyses. A $2 * 2$ repeated measure ANOVA was used to assess the effects of the EC condition (high EC group and low EC group) and directed forgetting ( $\mathrm{R}$ and $\mathrm{F}$ instruction). Trails with response times slower than $5 \mathrm{~s}$ or faster than $100 \mathrm{~ms}$ were excluded. $\mathrm{S}$ slower than $5 \mathrm{~s}$ or faster than $100 \mathrm{~ms}$ were excluded.

\section{RESULTS}

The statistical analysis software used is spss 22.0 . The results will show scores on the ATQ, accuracy and reaction time on the recognition test and perceptual identification test. The descriptive statistical results of accuracy are shown in Table 1a, and the reaction time is shown in Table $1 b$. 


\subsection{Screening of participants by $A T Q$}

Table 1. Descriptive Statistics for the Accuracy $(\mathrm{M} \pm \mathrm{SD})$

\begin{tabular}{ccccc}
\hline Variables & \multicolumn{2}{c}{ Recognition } & \multicolumn{2}{c}{ Perceptual identification } \\
& Remember & Forget & Remember & Forget \\
\hline High EC & $0.84 \pm 0.37$ & $0.58 \pm 0.49$ & $0.77 \pm 0.42$ & $0.63 \pm 0.49$ \\
Low EC & $0.73 \pm 0.44$ & $0.58 \pm 0.49$ & $0.82 \pm 0.39$ & $0.71 \pm 0.45$ \\
\hline
\end{tabular}

Table 2. Descriptive Statistics for the Reaction time (s) $(\mathrm{M} \pm \mathrm{SD})$

\begin{tabular}{cccc}
\hline Variables & Recognition & \multicolumn{2}{c}{ Perceptual identification } \\
& Remember & Forget & Remember \\
\hline High EC & $1.09 \pm 0.63$ & $1.26 \pm 0.75$ & $1.34 \pm 1.00$ \\
Low EC & $1.13 \pm 0.75$ & $1.11 \pm 0.67$ & $0.99 \pm 0.56$ \\
\hline
\end{tabular}

A total of 210 questionnaires were collected in this experiment, and 196 were left after the unqualified questionnaires were eliminated $(\mathrm{M}=4.47, \mathrm{SD}=0.83)$. There were 30 participates in the low EC group $(\mathrm{M}=$ $3.42, \mathrm{SD}=0.39$ ) and 19 participants in the high EC group $(\mathrm{M}=5.13, \mathrm{SD}=0.45)$. We conducted an independent-samples t-test $(\mathrm{t}=14.01, \mathrm{p}<0.001$, Cohen's $d=4.11$ ), and the results proved that our grouping was effective.

\subsection{The performance of $D F$ on explicit memory in individuals with different levels of EC}

A

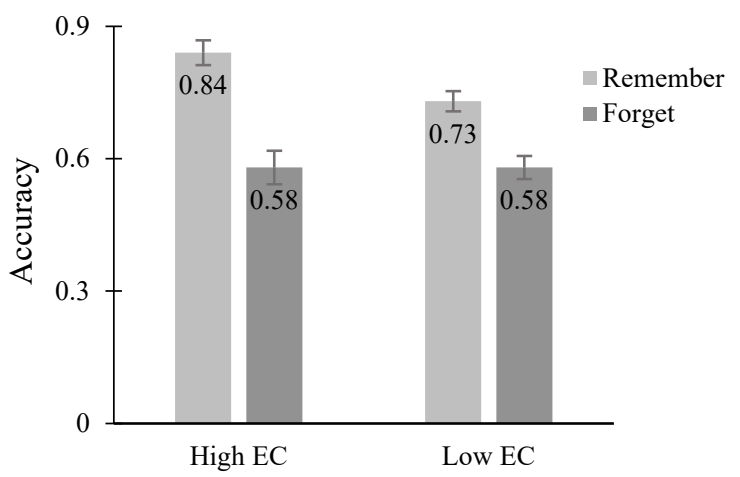

B

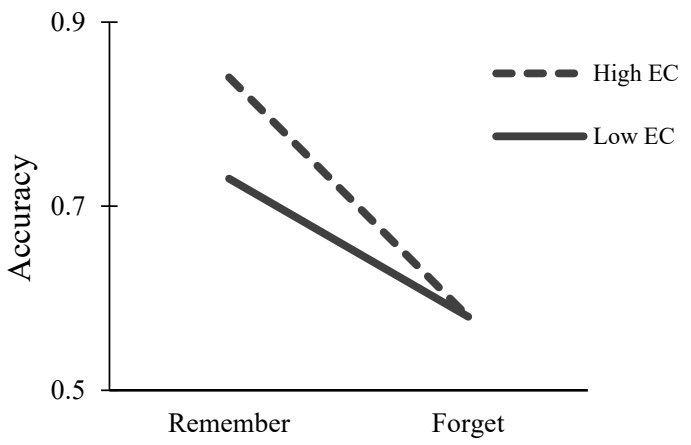

Figure 3 The accuracy of the recognition test. (A) A bar chart shows the level of EC on the X-axis and the accuracy on the $\mathrm{Y}$-axis with error lines based on standard error. (B) A line chart shows instructions type on the $\mathrm{X}$-axis and accuracy on the $\mathrm{Y}$-axis.
Firstly, after analysing the correct rate in the recognition experiment, the main effect of instruction is significant $\left(F=78.942, p<0.001, \eta^{2}=0.102\right)$. The accuracy of the characters given remembering instructions was significantly higher than that of the characters given forgetting instructions, which proved the existence of directed forgetting in explicit memory. The main effect of EC is also significant $(F=4.000, p=$ $\left.0.046, \eta^{2}=0.006\right)$. Individuals with high EC generally had higher recognition accuracy (Fig.3a). Most importantly, we found significant interactions $(\mathrm{F}=$ 5.879, $\left.\mathrm{p}=0.016, \eta^{2}=0.008\right)$. A simple effect analysis found that when given memory instructions, high EC had higher recognition accuracy $(F=11.717, p=0.001$, $\left.\eta^{2}=0.017\right)$. In addition, for individuals with low EC, the accuracy of giving memory instructions was significantly higher than that of giving forgetting instructions $\left(F=25.534, p<0.001, \eta^{2}=0.036\right)$, and the difference was even greater with high $\mathrm{EC}(\mathrm{F}=54.072, \mathrm{p}$ $<0.001, \eta^{2}=0.072$; Fig.3b).

The reaction time was also analysed (Fig.4a). The results show that the main effect of instruction is significant. $\left(F=3.993, p=0.046, \eta^{2}=0.006\right)$. The reaction time was longer to recognize the characters given the forgetting instruction. But no main effect of EC was found $\left(F=1.888, p=0.17, \eta^{2}=0.003\right)$. In addition, we also found significant interactions during the reaction time $\left(F=6.895, p=0.009, \eta^{2}=0.010\right)$. The results of further simple effect analysis showed that the reaction time was significantly longer in recognizing the characters given the forgetting instruction for high EC $\left(F=7.720, p=0.006, \eta^{2}=0.011\right)$. At the same time, the response time of high EC was significantly longer than that of low EC for the characters given forgetting order $\left(\mathrm{F}=9.008, \mathrm{p}=0.003, \eta^{2}=0.013\right)$. 
A

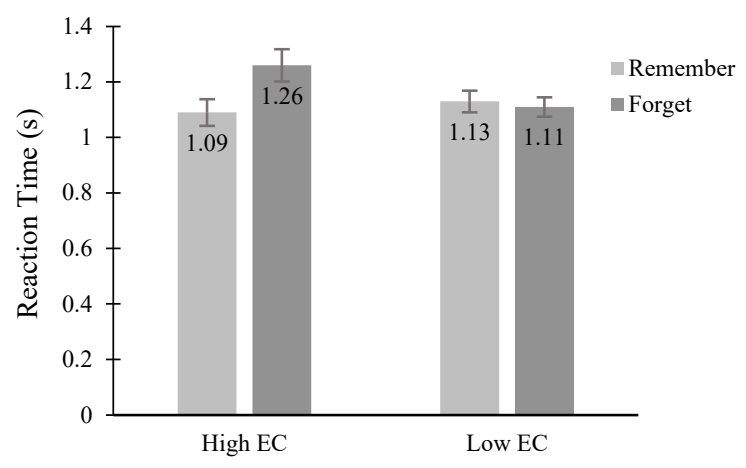

B

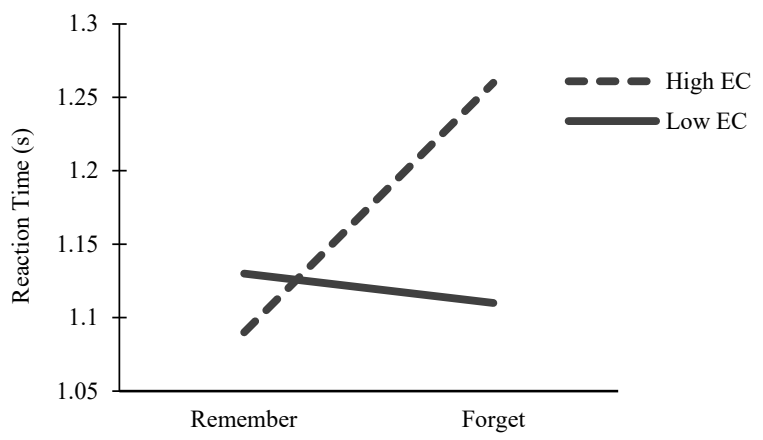

Figure 4 The Reaction Time of the recognition test. (A) A bar chart shows the level of EC on the X-axis and the reaction time on the $\mathrm{Y}$-axis with error lines based on standard error. (B) A line chart shows instructions type on the $\mathrm{X}$-axis and reaction time on the $\mathrm{Y}$-axis.

\subsection{The performance of DF on implicit memory in individuals with different levels of EC}

Following the same steps as the analysis of recognition results, it was found that the main effect of instruction was also significant in perceptual identification test $\left(F=13.604, p<0.001, \eta^{2}=0.037\right)$. This suggests that DF also exists in implicit memory (Fig.5). However, there is no interaction $(F=0.292, p=$ $\left.0.589, \eta^{2}=0.001\right)$ or main effect of EC $(F=3.436, p=$ $0.065, \eta^{2}=0.010$; Fig.5).

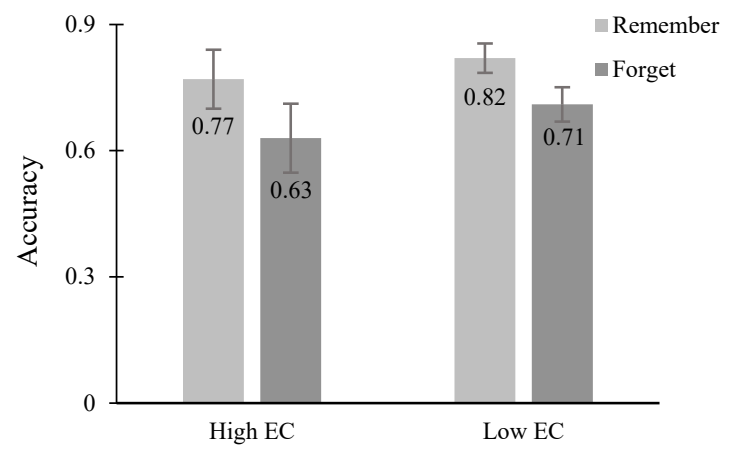

Figure 5 The accuracy of the perceptual identification test. A bar chart shows the level of EC on the X-axis and the accuracy on the $\mathrm{Y}$-axis with error lines based on standard error.
Then we further analysed the reaction time, we found that the main effect of EC was significant $(\mathrm{F}=$ $\left.26.849, \mathrm{p}<0.001, \eta^{2}=0.070\right)$, but the interaction $(\mathrm{F}=$ $\left.0.389, \mathrm{p}=0.533, \eta^{2}=0.001\right)$ and the main effect of instruction was not significant $\left(F=0.208, p=0.649, \eta^{2}\right.$ $=0.001)$. The results suggest that individuals with high EC had longer response times (Fig.6).

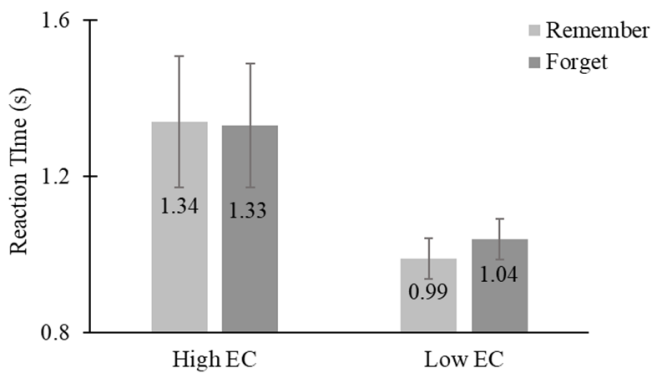

Figure 6 The Reaction Time of the perceptual identification test. A bar chart shows the level of EC on the $\mathrm{X}$-axis and the reaction time on the $\mathrm{Y}$-axis with error lines based on standard error.

\section{DISCUSSION}

Intend to understand better memory and forgetting, the present study aimed to re-examine the existence of DF in explicit and implicit memories and investigate whether it is affected by participants' effortful control level. Current data and statistical analysis obviously detected directed forgetting in explicit and implicit memories, which met previous expectations. But for the interaction between directed forgetting, memory type and EC level, outcomes were quite surprising. Firstly, for explicit memory, as expected, the characterremembering correct rate was within the forecast range, which proved a positive correlation between high EC scores and better-directed forgetting ability, vice versa. Nevertheless, mutual effect showed existence during explicit memory experiment, which showed that high EC participants needed longer RT when recognizing "forget" characters. It could be a manifestation of high $\mathrm{EC}$ as it basically equaled stronger inhibition ability. Therefore it took a longer time to recall. Secondly, although correct rate analysis helped to prove directed forgetting in implicit memory, no interaction or the main effect of EC was detected. RT analysis found the main effect of EC only, meaning that the high EC generally spent longer RT during the experiment. Overall, such findings may indicate that EC could influence explicit memory only, rather than affecting implicit memory as well. Moreover, the high EC may be more cautious and thoughtful, which results in longer RT.

The results were in agreement with some existing explanations in this field. Previously, some theories denied the significance of directed forgetting, simply concluding such forgetting as a result of lacking enough 
effective maintenance rehearsal so that information was not turned into STM successfully [14]. However, our findings were contrary to this rehearsal explanation according to the disparity between "remember" words and "forget" words recalling in the test phase. For this inconsistency, some research has proposed that directed forgetting could be approached by actively and subconsciously inhibitive cognition process [14], which is a possible explanation for our experiment results and conversely our results showed support for this theory.

The results are subject to certain limitations. For instance, a flaw of this study is that the formulated research aims could be too broad. Although we successfully managed to discover the existence of directed forgetting and if it is affected by EC in both explicit and implicit memories, the research may need to be narrowed down to have a better focus on a certain issue to generate deeper and specified conclusions. In addition, required by the independent variable of the study, the pre-screen of the experiment was a questionnaire on temperament, and several subjects selected to experiment participation showed interest in their questionnaire scores and ranking, thus may influencing their experiment performance due to test bias, which might be intensified as video calling was applied to control experiment conditions,

It is not a new opinion that the importance of forgetting is no less than that of remembering [1]. For more than 50 years, investigations of directed forgetting made it to be a technique within the researcher's grasp exploring memory updates under certain conditions [15]. Our results are significant to directed forgetting studies, and its newly exposed relationship with EC certainly showed more possibilities. The combination of findings is valuable to trauma research and coping as well as many related fields [16]. For example, it is vital in understanding memory and forgetting mechanisms of PTSD and a variety of mental disorders and could offer theoretical support to specific recovery treatment for patients who have distinct personality traits [17].

This would be a fruitful area for future work. Further research should be undertaken offline when it is safe to and apply neuroimaging techniques to better explore the neural basis behind the phenomena. Personality factors influencing cognition would also be an interesting area to investigate. Moreover, repeating such experiments in other cultures is of necessity as well.

\section{CONCLUSION}

In this research, the results have shown support to the existence of DF in both explicit and implicit memory and the crossing effect between cues and participants with high or low effortful control in Chinese adults. Although the measure to detect DF was quite traditional, it was novel to consider participants' effortful control level as a factor that may influence memory and forgetting. In spite of certain limitations such as wide topic and potential test bias, the results still provided a deeper insight to DF of explicit and implicit memory as well as its relation to a certain temperament, which are instrumental in mental disorder treatment and meaningful to the scope of cognitive psychology. More related brain function information is detected using neuroimaging technology and a greater investigation of factors affecting memory and forgetting would help to establish better accuracy on cognitive psychology. The results were in agreement with some existing explanations in this field. Previously, some theories denied the significance of directed forgetting, simply concluding such forgetting as a result of lacking enough effective maintenance rehearsal so that information was not turned into STM successfully. However, our findings were contrary to this rehearsal explanation according to the disparity between "remember" words and "forget" words recalling in the test phase. For this inconsistency, some research has proposed that directed forgetting could be approached by actively and subconsciously inhibitive cognition process, which is a possible explanation for our experiment results and conversely, our results showed support for this theory.

\section{REFERENCES}

[1] Basden, B. H. (1996). Directed Forgetting: Further Comparisons of the Item and List Methods. Memory, 4(6), 633-654. doi:10.1080/741941000.

[2] Brewin, C. R., Gregory, J. D., Lipton, M., \& Burgess, N. (2010). Intrusive images in psychological disorders: characteristics, neural mechanisms, and treatment implications. Psychology Review, 117(1), 210-232. doi:10.1037/a0018113.

[3] Chiu, Y. C., Wang, T. H., Beck, D. M., LewisPeacock, J. A., \& Sahakyan, L. (2021). Separation of item and context in item-method directed forgetting. Neuroimage, 235, 117983. doi:10.1016/j.neuroimage.2021.117983.

[4] Cottencin, O., Vaiva, G., Huron, C., Devos, P., Ducrocq, F., Jouvent, R., Thomas, P. (2006). Directed forgetting in PTSD: A comparative study versus normal controls. Journal of Psychiatric Research, $\quad 40(1), \quad 70-80$. doi:https://doi.org/10.1016/j.jpsychires.2005.04.00 1.

[5] Davis, R. L., \& Zhong, Y. (2017). The Biology of Forgetting-A Perspective. Neuron, 95(3), 490-503. doi:10.1016/j.neuron.2017.05.039.

[6] de Graaff, L. F., Honig, A., van Pampus, M. G., \& Stramrood, C. A. I. (2018). Preventing posttraumatic stress disorder following childbirth and 
traumatic birth experiences: a systematic review. Acta Obstet Gynecol Scand, 97(6), 648-656. doi:10.1111/aogs.13291.

[7] Diamond, A. (2013). Executive functions. Annual Review of Psychology, 64, 135-168. doi:10.1146/annurev-psych-113011-143750.

[8] Evans, D. E., \& Rothbart, M. K. (2007). Developing a model for adult temperament. Journal of Research in Personality, 41(4), 868-888. doi:10.1016/j.jrp.2006.11.002.

[9] Fawcett, J. M., Lawrence, M. A., \& Taylor, T. L. (2016). The representational consequences of intentional forgetting: Impairments to both the probability and fidelity of long-term memory. Journal of Experimental Psychology: General, 145(1), 56-81. doi:10.1037/xge0000128.

[10] Fawcett, J. M., \& Taylor, T. L. (2008). Forgetting is effortful: evidence from reaction time probes in an item-method directed forgetting task. Memory and Cognition, 36(6), 1168-1181. doi:10.3758/MC.36.6.1168.

[11] Geraerts, E., \& McNally, R. J. (2008). Forgetting unwanted memories: directed forgetting and thought suppression methods. Acta Psychologica (Amst), 127(3), 614-622. doi:10.1016/j.actpsy.2007.11.003.

[12] Lin, W.-L., Hsu, K.-Y., Chen, H.-C., \& Chang, W.y. (2013). Different attentional traits, different creativities. Thinking Skills and Creativity, 9, 96106. doi:10.1016/j.tsc.2012.10.002.

[13] Derryberry, D., \&Rotherbart, M. K. (1988). Arousal, affect, and attention as components of temperament. Journal of Personality and Social Psychology, 55(6), 958-966. doi: 10.1037/00223514.55.6.958.

[14] MacLeod, C. M. (2012). Directed Forgetting. In N. M. Seel (Ed.), Encyclopedia of the Sciences of Learning (pp. 993-995). Boston, MA: Springer US.

[15] Ren, X., Li, Y., Mao, W., \& Geng, Q. (2019). The effect of emotion on directed forgetting for continuous events. Acta Psychologica Sinica, 51(3). doi:10.3724/sp.J.1041.2019.00269.

[16] Rothbart, M. K., Ahadi, S. A., Hershey, K. L., \& Fisher, P. (2001). Investigations of temperament at three to seven years: the Children's Behavior Questionnaire. Child Development, 72(5), 13941408. doi:10.1111/1467-8624.00355.

[17] Wang, T. H., Placek, K., \& Lewis-Peacock, J. A. (2019). More Is Less: Increased Processing of Unwanted Memories Facilitates Forgetting. Journal of Neuroscience Methods, 39(18), 3551-3560. doi:10.1523/JNEUROSCI.2033-18.2019. 